\title{
AN INFINITE FAMILY OF DISTINCT 7-MANIFOLDS ADMITTING POSITIVELY CURVED RIEMANNIAN STRUCTURES
}

\author{
BY SIMON ALOFF AND NOLAN R. WALLACH ${ }^{1}$
}

Communicated by S. S. Chern, February 24, 1974

1. Introduction. The purpose of this note is to show that if $T^{\mathbf{1}}$ is a closed, connected one-dimensional subgroup of $S U(3)$ that has no nonzero fixed points, then $S U(3) / T^{1}$ admits an $S U(3)$-invariant Riemannian structure of strictly positive curvature. This result implies the statement of the title since there are an infinite number of distinct homotopy types among the spaces $S U(3) / T^{\mathbf{1}}$ with $T^{\mathbf{1}}$ as above (see Lemma 3.3).

To prove the above result we introduce what we call condition II, which generalizes condition III of [2] and [3]. Although the spaces $S U(3) / T^{1}$ are the only new spaces satisfying condition II (see Theorem 5.1 below), it is worthwhile to introduce the notion since the 13-dimensional example, $M_{2}$, of Berger [1] satisfies condition II in a nontrivial fashion. Hence there is a set of invariant metrics $\langle,\rangle_{t}$ on $M_{2}$ with $-1<t<\frac{1}{3}$ of strictly positive curvature (see $\S 4$ ) with $\langle,\rangle_{0}$ the only normal one.

A word should be said about the joint authorship of this paper. The main result of this note was found independently by the authors by slightly different methods. We have given the technique of the second author since it is conceptually simpler. Lemma 3.3 was pointed out to the second author by Professor Lashof of the University of Chicago and derived independently by the first author.

The second author would like to also thank Professor M. P. do Carmo and the students of IMPA, Rio de Janeiro, for their help during his research on this problem.

2. Condition II. Let $G$ be a compact connected Lie group and let $K$ be a closed subgroup of $G$.

Definition 2.1. ( $G, K)$ is said to satisfy condition II if there is an $\operatorname{Ad}(G)$-invariant inner product $\langle,\rangle_{0}$ on $\mathfrak{g}$, the Lie algebra of $G$, such

AMS (MOS) subject classifications (1970). Primary 53C20, 53C30.

${ }^{1}$ Research partially supported by a Sloan Fellowship, by the NSF and by the O.A.S. 
that if $\mathfrak{1}$ is $\mathfrak{p}$ ( $\mathfrak{f}$ the Lie algebra of $K$ ) then:

(1) $\mathfrak{p}=V_{1} \oplus V_{2}$ orthogonal direct sum, $\operatorname{Ad}(K) V_{i} \subset V_{i}, i=1,2$.

(2) $\left[V_{1}, V_{2}\right] \subset V_{2}$.

(3) $\left[V_{1}, V_{1}\right] \subset \mathfrak{f}+V_{1}$.

(4) $\left[V_{2}, V_{2}\right] \subset \mathfrak{f}+V_{1}$.

(5) If $x=x_{1}+x_{2}, y=y_{1}+y_{2}, x_{i}, y_{i} \in V_{i}, i=1,2$, and if $[x, y]=0$, $x \wedge y \neq 0$ then $\left[x_{1}, y_{1}\right] \neq 0$.

In [2] and [3] we introduced condition III. Condition III implies condition II.

LemmA 2.2. Let $(G, K)$ satisfy (1), (2), (3), (4) of condition II. Let the notation be as in Definition 2.1. Let $-1<t<\infty$ be given and let $\langle$, be the inner product on $\mathfrak{p}$ given by

$$
\left\langle x_{1}+x_{2}, y_{1}+y_{2}\right\rangle=(1+t)\left\langle x_{1}, y_{1}\right\rangle_{0}+\left\langle x_{2}, y_{2}\right\rangle_{0}
$$

for $x_{i}, y_{i} \in V_{i}$. Let $R$ be the curvature tensor of the corresponding Riemannian structure on $G / K$. If we identify $T(G / K)_{e K}$ with $\mathfrak{p}$ then

$$
\begin{aligned}
\langle R(x, y) y, x\rangle= & \frac{1-3 t}{4}\left\langle[x, y]_{1},[x, y]_{1}\right\rangle_{0} \\
& +\left(t-t^{2}\right)\left\langle\left[x_{1}, y_{1}\right]_{1},[x, y]_{1}\right\rangle_{0}+t^{2}\left\langle\left[x_{1}, y_{1}\right]_{1},\left[x_{1}, y_{1}\right]_{1}\right\rangle_{0} \\
& +\frac{(1+t)^{2}}{4}\left\langle[x, y]_{2},[x, y]_{2}\right\rangle+\left\langle[x, y]_{\mathfrak{t}},[x, y]_{\mathfrak{t}}\right\rangle_{0} \\
& +\left(t-t^{2}\right)\left\langle\left[x_{1}, y_{1}\right]_{\mathfrak{t}},[x, y]_{\mathfrak{t}}\right\rangle_{0}+t^{2}\left\langle\left[x_{1}, y_{1}\right]_{\mathfrak{t}},\left[x_{1}, y_{1}\right]_{\mathfrak{t}}\right\rangle_{0},
\end{aligned}
$$

where if $Z \in \mathfrak{g}, Z=Z_{\mathfrak{t}}+Z_{1}+Z_{2}, Z_{\mathfrak{t}} \in \mathfrak{f}, Z_{1} \in V_{1}, Z_{2} \in V_{2}$.

The proof of this lemma is essentially the same as that of Lemma 7.3 of [3] (here one uses the proof of Lemma 7.1 of [3] which goes through unchanged from condition III to condition II).

THEOREM 2.4. If $(G, K)$ satisfies condition II, then the $G$-invariant Riemannian structures on $G / K$ corresponding to $-1<t<0$ in Lemma 2.3 have strictly positive curvature. If in condition II (Definition 2.1), (3) is

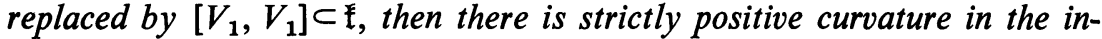
tervals $-1<t<0$ and $0<t<\frac{1}{3}$.

Proof. Let $\|x\|^{2}=\langle x, x\rangle_{0}, x \in \mathfrak{g}$. Let $a=\left\|[x, y]_{1}\right\|, b=\left\|\left[x_{1}, y_{1}\right]_{1}\right\|$, $c=\left\|[x, y]_{2}\right\|, d=\left\|[x, y]_{t}\right\|, \quad e=\left\|\left[x_{1}, y_{1}\right]_{t}\right\|$. Lemma 2.3 implies that if $x, y \in \mathfrak{p}$ then

$$
\begin{aligned}
\langle R(x, y) y, x\rangle \geqq & \left(\frac{1-3 t}{4}\right) a^{2}-\left|-t^{2}\right| a b+t^{2} b^{2} \\
& +\frac{(1+t)^{2}}{4} c^{2}+d^{2}-\left|t-t^{2}\right| d e+t^{2} e^{2}
\end{aligned}
$$


Let

$$
\begin{aligned}
& Q_{1}(a, b)=\left(\frac{1-3 t}{4}\right) a^{2}-\left|t-t^{2}\right| a b+t^{2} b^{2} \\
& Q_{2}(d, e)=d^{2}-\left|t-t^{2}\right| d e+t^{2} e^{2} .
\end{aligned}
$$

A computation of determinants shows that $Q_{1}$ is positive definite if $-1<t<0$, and $Q_{2}$ is positive definite if $-1<t<0,0<t<\frac{1}{3}$. Thus since

$$
\langle R(x, y) y, x\rangle \geqq Q_{1}(a, b)+Q_{2}(d, e)+\frac{(1+t)^{2}}{4} c^{2}
$$

if $\langle R(x, y) y, x\rangle=0$, and $-1<t<0$ then $a=b=c=d=e=0$. But then $[x, y]=0,\left[x_{1}, y_{1}\right]=0$. Thus $x \wedge y=0$. If $\left[V_{1}, V_{1}\right] \subset \mathfrak{z}$. Then $Q_{1}(a, b)=$ $((1-3 t) / 4) a^{2}$. Thus

$$
\langle R(x, y) y, x\rangle \geqq\left(\frac{1-3 t}{4}\right) a^{2}+\frac{(1+t)^{2}}{4} c^{2}+Q_{2}(d, e) .
$$

Hence if $x \wedge y \neq 0,\langle R(x, y), y, x\rangle>0$ if $-1<t<0,0<t<\frac{1}{3}$. Q.E.D.

3. $S U(3) / T^{1}$. Let $G=S U(3)$. Then every nontrivial circle in $S U(3)$ is of the form

$$
T_{k, l}=\left\{\left[\begin{array}{ccc}
e^{2 \pi i k \theta} & 0 & 0 \\
0 & e^{2 \pi i l \theta} & 0 \\
0 & 0 & e^{-2 \pi i(k+l) \theta}
\end{array}\right] \mid \theta \in \boldsymbol{R}\right\}, \quad|k|+|l| \neq 0,
$$

$k, l \in Z$, up to conjugacy in $S U(3)$. Let

$$
G_{1}=\left\{\left[\begin{array}{cc}
g & 0 \\
0 & \operatorname{det} g^{-1}
\end{array}\right] \mid g \in U(2)\right\} .
$$

Then $T_{k, l} \subset G_{1}$. Let $g_{1}$ be the Lie algebra of $G_{1}$ in $\mathfrak{g}$, the Lie algebra of $G$. Take $\langle X, Y\rangle_{0}=-\operatorname{Re}(\operatorname{tr} X Y), X, Y \in \mathrm{g}$. Then

$$
\mathrm{g}_{1}^{\perp}=\left\{\left[\begin{array}{cc}
0 & z \\
t_{\bar{z}} & 0
\end{array}\right] \mid z=\left[\begin{array}{l}
z_{1} \\
z_{2}
\end{array}\right], z_{i} \in C\right\} .
$$

Take $V_{2}=\mathfrak{g}_{1}^{\perp}$. Let

$$
h_{k, l}=\left[\begin{array}{ccc}
2 \pi i k & & 0 \\
0 & 2 \pi i l & 0 \\
0 & & -2 \pi i(l+k)
\end{array}\right] .
$$

Take $V_{1}=h_{k, l}^{\perp} \cap g_{1}$.

Since ( $\left.S U(3), G_{1}\right)$ is the symmetric pair corresponding to $C P^{2}$, we see that if $x, y \in V_{2}$ and $[x, y]=0$ then $x \wedge y=0$. A computation shows that if $x, y \in V_{1}$ and $[x, y]=0$, then $x \wedge y=0$ (we are assuming $|k|+|l| \neq 0$ ). 
If $k l>0$ one sees by a direct computation of determinants that if $x \in V_{1}$, $y \in V_{2}$ and $[x, y]=0$ then $x=0$ or $y=0$.

LEMMA 3.1. If $|k|+|l| \neq 0$ and $k l>0$ then $\left(S U(3), T_{k, l}\right)$ satisfies condition II.

Proof. Conditions (1), (2), (3), (4) are clearly satisfied.

Suppose $x=x_{1}+x_{2}, y=y_{1}+y_{2}, x_{i}, y_{i} \in V_{i}, i=1,2$, and that $[x, y]=0$, $\left[x_{1}, y_{1}\right]=0$ and $x \wedge y \neq 0$.

If $x_{1}=0$. Then $0=[x, y]=\left[x_{2}, y_{2}\right]+\left[x_{2}, y_{1}\right]$. But $\left[x_{2}, y_{2}\right] \in \boldsymbol{R} h_{k, l}+V_{1}$, $\left[x_{2}, y_{1}\right] \in V_{2}$. Thus $\left[x_{2}, y_{1}\right]=0$. Hence since $x \neq 0, y_{1}=0$. But $\left[x_{2}, y_{2}\right]=0$ implies $0=x_{2} \wedge y_{2}=x \wedge y$. Hence $x_{1} \neq 0$. If $x_{2}=0$ then $\left[x_{1}, y_{2}\right]=0$ thus $y_{2}=0$ and $x_{1} \wedge y_{1}=0$. Thus $x_{2} \neq 0$. Finally, $[x, y]=0$ implies $\left[x_{1}, y_{1}\right]+\left[x_{2}, y_{2}\right]=0$. Thus since $\left[x_{1}, y_{1}\right]$ is assumed to be zero $\left[x_{2}, y_{2}\right]=0$. Thus $y_{2}=\lambda x_{2}$, $y_{1}=\mu x_{1}, \lambda, \mu \in R$. But

$$
0=\left[x_{1}, y_{2}\right]+\left[x_{2}, y_{1}\right]=\lambda\left[x_{1}, x_{2}\right]+\mu\left[x_{2}, x_{1}\right]=(\lambda-\mu)\left[x_{1}, x_{2}\right] .
$$

Since $\left[x_{1}, x_{2}\right] \neq 0, \lambda=\mu$. Thus $\lambda x=y$ contradicting $x \wedge y \neq 0$.

THEOREM 3.2. Let $T^{1}$ be a closed one-dimensional subgroup of $S U(3)$, such that there is no $v \neq 0$ in $C^{3}$ so that $T^{1} v=v$. Then $S U(3) / T^{1}$ has an $S U(3)$-invariant Riemannian structure of strictly positive curvature.

Proof. $\quad T^{1} \subset S U(3)$ is conjugate to $T_{k, l}$ with $|k|+|l| \neq 0$.

We note that $T_{k, l}$ is conjugate to $T_{l, k}, T_{k,-l-k}$ and $T_{l,-l-k}$ in $S U(3)$. If $k l>0$ the result follows from Lemma 3.1 and Theorem 2.4. Thus we may assume $k l<0$. Since $T_{k, l}, T_{k,-l-k}$ and $T_{l,-l-k}$ are conjugate we may assume $k>0, l<0$.

(1) $|l|<k$. Then $l(-l-k)=-l^{2}-l k>l^{2}-l^{2}=0$. Thus, $T_{l,-l-k}$ satisfies condition II. Since $T_{l,-l-k}$ is conjugate to $T_{k, l}, G / T_{k, l}=G / T_{l,-l-k}$ as a $G$-space.

(2) $|l|>k$. Then $k(-l-k)=-k l-k^{2}>k^{2}-k^{2}=0$. We can therefore argue as in (1). The result now follows. Q.E.D.

LEMMA 3.3. Suppose that $k, l$ are relatively prime. Then

$$
H^{4}\left(S U(3) / T_{k, l}, Z\right)=Z / r Z
$$

with $r=\left|k^{2}+l^{2}+k l\right|$

4. Berger's example $M_{2^{\bullet}}$ Let $S U(5) \supset S p(2) \times T^{1}$ as in Berger [1]. Then using the computations of [1], one finds that $\left(S U(5), S p(2) \times T^{1}\right)$ satisfies condition II with $V_{2} \neq 0$ and $\left[V_{1}, V_{1}\right] \subset \mathfrak{f}$. The metrics $\langle,\rangle_{t}$ of Theorem 2.4 are not normal for $-1<t<0,0<t<\frac{1}{3}$. 


\section{The classification.}

THEOREM 5.1. If $(G, K)$ satisfies condition II then one of the following is true.

(1) $G / K$ has positive curvature relative to a normal ( $t=0$ in Lemma 2.3) Riemannian structure (take $V_{2}=0$ in condition II).

(2) $(G, K)$ satisfies condition III.

(3) $G=S U(3)$ or $U(3)$ and $G / K$ is covered by $S U(3) / T^{1}$.

Note. Berger [1] has classified all $(G, K)$ in (1) above. Also (3) says that the exceptional examples of [3, Corollary 6.2] have metrics of positive curvature. Thus to classify the $G / K$ admitting $G$-invariant metrics of positive curvature, one may assume that $G$ is semisimple.

Proof (OUtline). Let $\mathfrak{g}=\mathfrak{f} \oplus V_{1} \oplus V_{2}$ as in condition II. Let $\mathfrak{g}_{1}=\mathfrak{f} \oplus V_{1}$. Let $G_{1}$ be the connected subgroup of $G$ corresponding to $g_{1}$. Let $Z$ be the identity component of the center of $G(\operatorname{dim} Z \leqq 1$ by [3, Corollary 4.2]). Then condition II implies $G_{1} \supset Z$ and

(a) $\left(G / Z, G_{1} / Z\right)$ is a symmetric pair of compact type corresponding to a rank 1 symmetric space.

(b) $\left(G_{1}, K\right)$ is positively curved relative to a normal Riemannian structure.

(a) implies $G / Z$ is locally isomorphic with $S U(n), S O(n), S p(n)$ or $F_{4}$ and that $\left(G_{1}, K\right)$ is (up to the center of $\left.G_{1}\right)$ one of the pairs of Berger's classification in [1]. The result follows by a case by case check.

\section{REFERENCES}

1. M. Berger, Les variétés riemanniennes homogènes normales simplement connexes a courbure strictement positive, Ann. Scuola Norm. Sup. Pisa (3) 15 (1961), 179-246. MR 24 \#A2919.

2. N. R. Wallach, Three new examples of compact manifolds admitting Riemannian structures of strictly positive curvature, Bull. Amer. Math. Soc. 78 (1972), 55-56.

3. - Compact homogeneous Riemannian manifolds with strictly positive curvature, Ann. of Math. (2) 96 (1972), 277-295. MR 46 \#6243.

Instituto de Matemática Pura e Aplicada, Rio de Janeiro, Brasil

Department of Mathematics, University of Chicago, Chicago, Illinois 60637

Department of Mathematics, Rutgers University, New Brunswick, New Jersey 08903 